\title{
Core Poloidal Rotation and Internal Transport Barrier Formation in TFTR
}

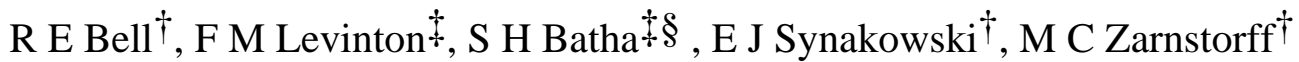 \\ $\dagger^{\dagger}$ Princeton Plasma Physics Laboratory, Princeton, New Jersey 08543 \\ \$Fusion Physics and Technology, Torrance, California 90503
}

\begin{abstract}
Impurity poloidal rotation velocities have been measured in the core of TFTR plasmas using a new spectroscopic diagnostic. Two types of transitions to enhanced confinement in reversed shear plasmas are examined. A bifurcation in carbon poloidal rotation is observed to occur before the transition to enhanced confinement for one of these types, while other measured plasmas parameters remain constant. A narrow radial region with reversed poloidal rotation and rotational shear is established 60-100 ms before the transition, and is associated with a large negative radial electric field.
\end{abstract}

\section{Introduction}

Tokamak plasmas with negative central magnetic shear have exhibited desirable confinement characteristics within a core transport barrier (Levinton et al 1995, Strait et al 1995). A complete understanding of the dynamics of barrier formation requires accurate measurements of all quantities in the radial force balance equation,

$$
E_{r}=\nabla p /(e Z n)+v_{\phi} B_{\theta}-v_{\theta} B_{\phi}
$$

where $p$ is the ion pressure, $e$ is the electronic charge, $Z$ is the charge number, $n$ is the ion density, $v_{\phi}$ and $v_{\theta}$ are the toroidal and poloidal velocity, $B_{\phi}$ and $B_{\theta}$ are the toroidal and poloidal magnetic fields. The carbon pressure and toroidal velocity have routinely been measured on TFTR using charge exchange recombination spectroscopy. Presented here are

$\S$ Present address: Science Research Laboratory 15 Ward St. Somerville, MA 02144 
new measurements of carbon $v_{\theta}$ profiles and $E_{\mathrm{r}}$ in the core of TFTR plasmas and their connection to the formation of transport barriers in enhanced reversed shear (ERS) plasmas.

In TFTR ERS plasmas, two types of transitions to improved confinement have been observed (Bell et al 1996). Though they share improvements in particle, ion thermal and momentum confinement, they differ significantly in power threshold $(\approx 20 \mathrm{MW}$ for Type I, $\leq 5 \mathrm{MW}$ for Type II). Type I transitions show a sudden increase $\partial \mathrm{n}_{\mathrm{e}} / \partial \mathrm{t}$ within the core, while Type II transitions are marked by an abrupt increase in $\mathrm{T}_{\mathrm{i}}$ and $\mathrm{T}_{\mathrm{e}}$. Type I transitions, using neoclassically calculated values of $v_{\theta}$, are consistent with $E \times B$ shear flow suppression of turbulence as a triggering mechanism (Bell et al 1996, Synakowski et al 1997). Type II transitions were found to occur with a variety of $\mathrm{E}_{\mathrm{r}}$ profiles, suggesting no clear link to $E \times B$ shear flow as a trigger mechanism. Despite the low power threshold, Type II transitions were only observed late in the discharge at similar times when $q_{\text {min }} \geq$ 2, suggesting a link with the evolving current density profile.

\section{Experimental}

The $v_{\theta}$ measurements were made using a new spectroscopic diagnostic designed to measure carbon poloidal velocities in the core of TFTR plasmas using the Doppler shift of

the C VI $5291 \AA$ impurity line. The diagnostic had a $20 \mathrm{~ms}$ time resolution and a radial resolution of $\leq 3.5 \mathrm{~cm}$ after an inversion (Bell 1997) is applied. All $v_{\theta}$ data shown here are chord-averaged. The motional Stark effect (MSE) diagnostic (Levinton et al 1989), which measures the pitch angle of the magnetic field lines, is also sensitive to $E_{r}$ (Zarnstorff et al 1997).

During the startup of Type I ERS plasmas, a current ramp was used with early low power neutral beam injection to produce a hollow current profile. Higher neutral beam 
power was then added in a heating phase. Lithium pellet injection, which has proven to be useful in achieving conditions favorable for a transition, was used during the early low power phase. Typical parameters were $R_{0}=260 \mathrm{~cm}$, and $B_{T}=4.6,3.4 \mathrm{~T}$. The higher field is typical for most TFTR ERS discharges. The neutral beam power during the heating phase, $P_{N B}$, was typically 25-28 MW for high $B_{T}$ discharges, and 13-15 MW for low $B_{T}$ discharges. The plasma current $I_{\mathrm{p}}=1.6 / 1.2 \mathrm{MA}$ was used in the high/low field discharges, which were set up to have nominally the same edge $q$.

The startup of ERS plasma with a Type II transitions was similar with $B_{\mathrm{T}}=4.8 \mathrm{~T}$, $I_{p}=1.6 \mathrm{MA}, P_{N B}=13 \mathrm{MW}$. The Li pellet was injected at the start of the heating phase.

\section{Results}

Two reversed shear discharges are seen to undergo a bifurcation in confinement shortly after the start of the high power heating phase, as indicated by the carbon pressure in Fig. 1a. Shown in Fig. 1b is the chord-averaged carbon $v_{\theta}$ for these two discharges, which diverged before the transition. The carbon ions at this location which had been rotating in the positive (ion diamagnetic) direction began a reversal in direction about $60 \mathrm{~ms}$ before the first indication of a confinement improvement. The excursion in rotation peaks and then relaxed as the confinement improvement took place. This change in $v_{\theta}$ was radially localized, typically appearing on only one or two viewing chords.

Much larger changes in the $v_{\theta}$ precursor are sometimes seen in the lower field experiments $\left(\mathrm{B}_{\mathrm{T}}=3.4 \mathrm{~T}\right)$. Figures 2 and 3 show the chord-averaged $v_{\theta}$ in a narrow radial region change by more than $40 \mathrm{~km} / \mathrm{s}$ prior to the ERS transition. The full magnitude of the negative velocity is reduced by the chord averaging of the data. This large negative $v_{\theta}$ corresponds to a large negative $E_{r}$. Shown in Fig. $2 \mathrm{~b}$ is the change in $E_{r}$ as determined by the MSE diagnostic; the change is $E_{r}$ is so large, localized, and rapid that its contribution to 
the measured polarization angle is readily separable from the slowly changing magnetic field contribution. There is a strong temporal and spatial correlation between the measured $v_{\theta}$ and the measured change in $E_{r}$.

Two poloidal velocity profiles are plotted against the tangency radii of the sightlines in Fig. 3. The large negative well that develops in the poloidal velocity profile is narrower than it appears. Sightlines interior to the shear layer include diminishing samples of the negative velocity region. The radial extent of this high velocity shear region was small compared to the spatial separation of the $v_{\theta}$ sightlines $(3.5 \mathrm{~cm})$ or the MSE sightlines (4 $\mathrm{cm})$. Comparison of the $v_{\theta}$ profile and the measured $q$ profile in Fig. 3 indicates that the location of the negative velocity region was a few centimeters inside of the radial location of the minimum in the safety factor, $q_{\min }$.

Poloidal rotation measurements for a Type II ERS transition are shown in Fig. 4. The central ion temperature $T_{i}(0)$ increased suddenly by $60 \%$ at the transition. There was a smaller increase in $T_{e}$, with little change in $n_{e}$ or $v_{\phi}$. The electrons are presumably being heated by the ions, since, like the Type I ERS plasmas, there is no apparent improvement in the electron thermal conductivity after the transition. No change in $v_{\theta}$ was seen before the transition. After the transition, $v_{\theta}$ increased, consistent with the increase in $\nabla p$ due to the increased $T_{i}$. Using the inversion point of the normalized heating rate $\left(1 / T_{e} d T_{e} / d t\right)$ from ECE measurements, for similar Type II discharges, the location of the transport barrier was found to be a few centimeters outside of the location of $q_{\text {min }}$. (see Fig. 4 in Bell et al 1996)

\section{Discussion}

The proposed role of $E \times B$ shear in the formation of the transport barrier is reinforced for the Type I ERS transition by the new core poloidal velocity measurements. 
At least for one discharge, though, no poloidal velocity precursor was observed before the ERS transition, indicating perhaps that the observed poloidal excursion is not a necessary condition for a transition. There is no clear generating mechanism for the poloidal excursion and negative $E_{r}$ at this time. There are remarkable similarities between the linked behavior of $v_{\theta}$ and $E_{r}$ in the core to those observed at L-H transitions at the edge. Some of the proposed mechanisms for the generation of the $E_{r}$ at the edge warrant investigating in the core.

The neoclassical calculations of the poloidal velocity, previously used on TFTR to evaluate the role $E \times B$ shear played in the formation and sustainment of the transport barrier, of course, could not anticipate the dynamics of the large poloidal velocity and the velocity shear observed. Differences between measured and neoclassically calculated $v_{\theta}$ may explain the differences in the calculated turbulence growth rate and the shearing rate at the time of transition for low field discharges (Levinton et al 1996). This will be addressed in future work.

The differences in the power threshold, the timing, the poloidal rotation, and the location of the barrier at transition suggest the Type II transition has a different triggering mechanism than the Type I transition.

\section{Acknowledgement}

This work has been supported by the US Department of Energy Contract No. DEAC02-76-CH03073. 


\section{References}

Bell R E et al 1996 Proc. 23rd EPS Conference on Controlled Fusion and Plasma Physics (Kiev) 20C Part I p 59

Bell R E 1997 Rev. Sci. Instrum. 681273

Levinton F M et al 1989 Phys. Rev. Lett. 632060

Levinton F M et al 1995 Phys. Rev. Lett. 754417

Levinton F M et al 1996 Proc. 16th International Conference on Fusion Energy (Montreal, 1996) (IAEA, Vienna, 1997) IAEA-CN-64/A1-3 Vol. 1 p 211

Strait E J et al 1995 Phys. Rev. Lett. 754421

Synakowski E J et al 1997 Phys. Plasmas 41737

Zarnstorff M C et al 1997 Phys. Plasmas 41097 

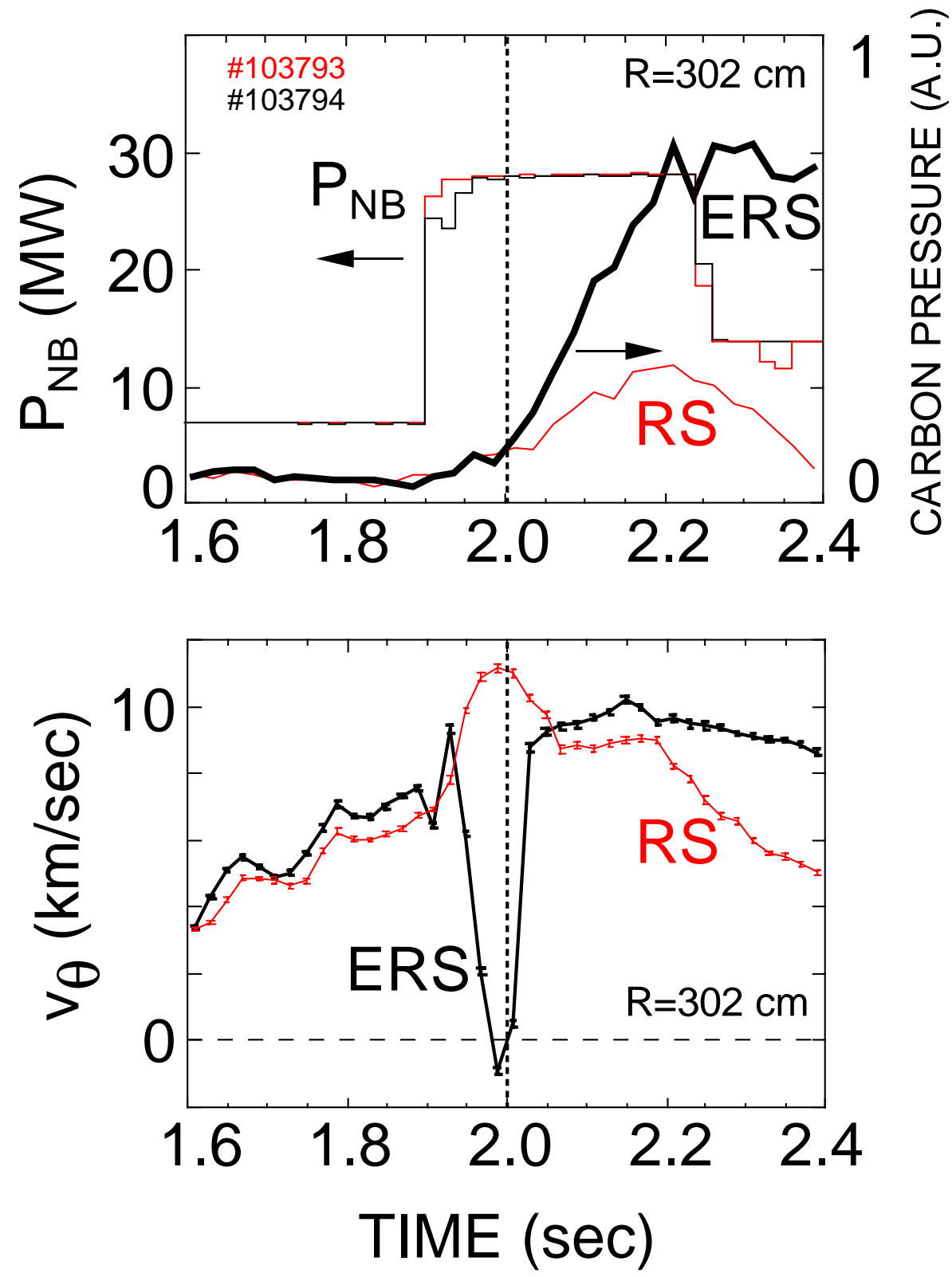

Fig. 1 Comparison of two reversed shear discharges. (a) The ERS transition time is indicated by the bifurcation in the local carbon pressure. (b) A bifurcation in the carbon poloidal velocity occurs before the transport improvement. Positive values of $v_{\theta}$ are in the ion diamagnetic drift direction. 

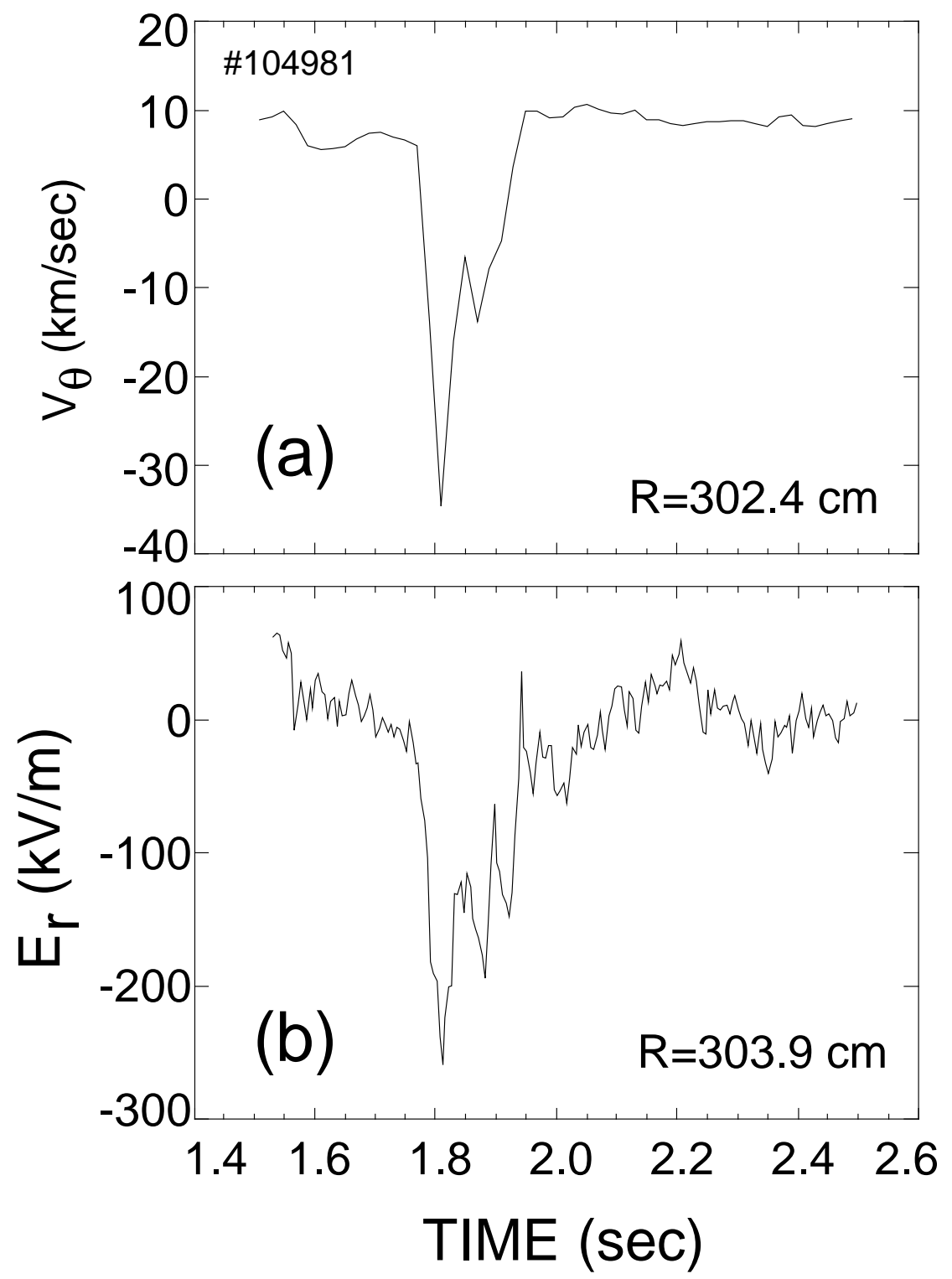

Fig. 2 Measured carbon $v_{\theta}$ and $E_{r}$ show similar time behavior. (a) A large excursion in $v_{\theta}$ is observed in a low field $\left(B_{T}=3.4 \mathrm{~T}\right)$ ERS discharge on a single sightline (b) Change in $E_{r}$ measured by MSE diagnostic 


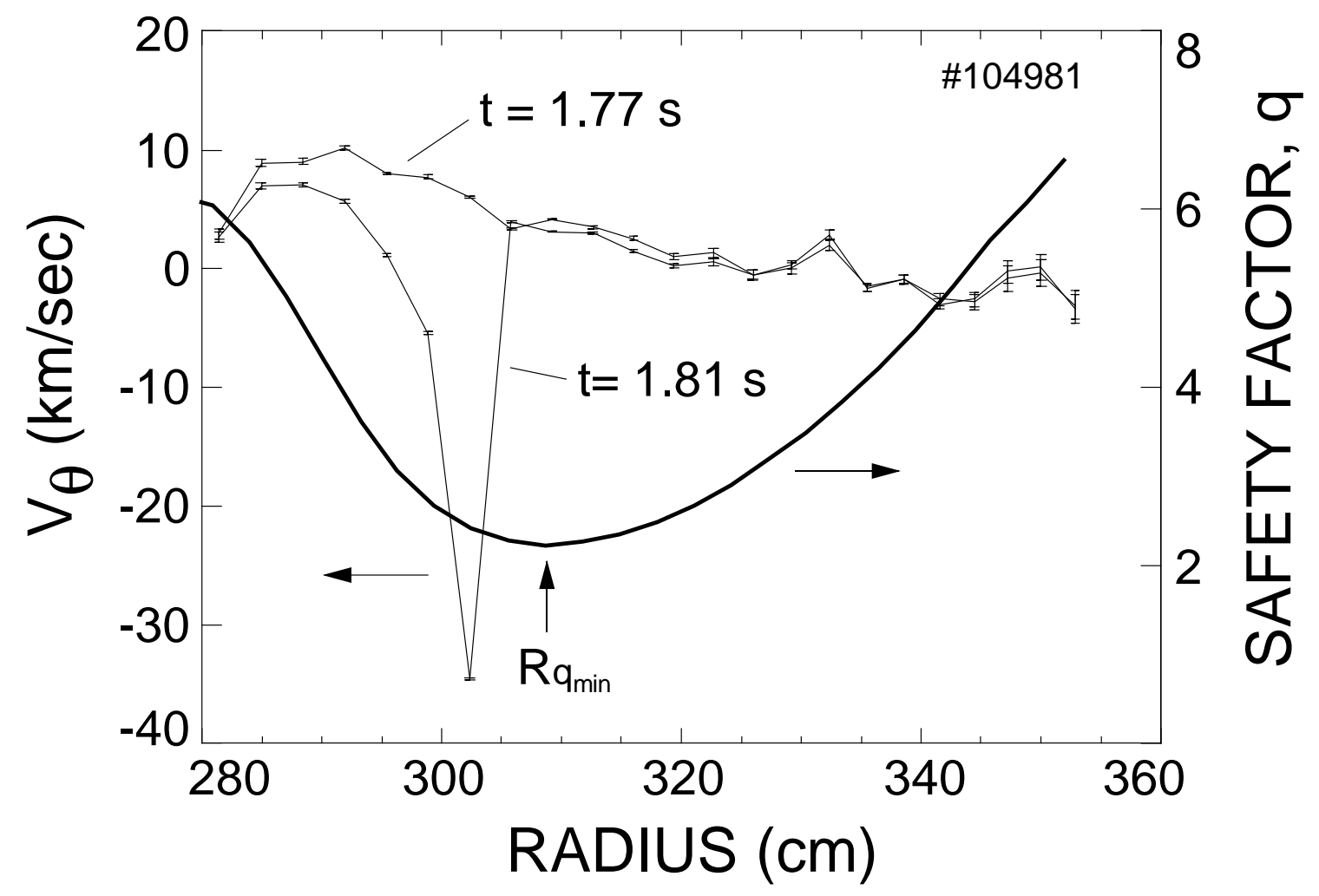

Fig. 3 Carbon poloidal rotation profiles at two times before the ERS transition, before and during the local excursion in $v_{\theta}$. Overlaid is the measured $q$ profile. The location of the velocity shear layer is just inside of the location of $q_{m i n}$. 

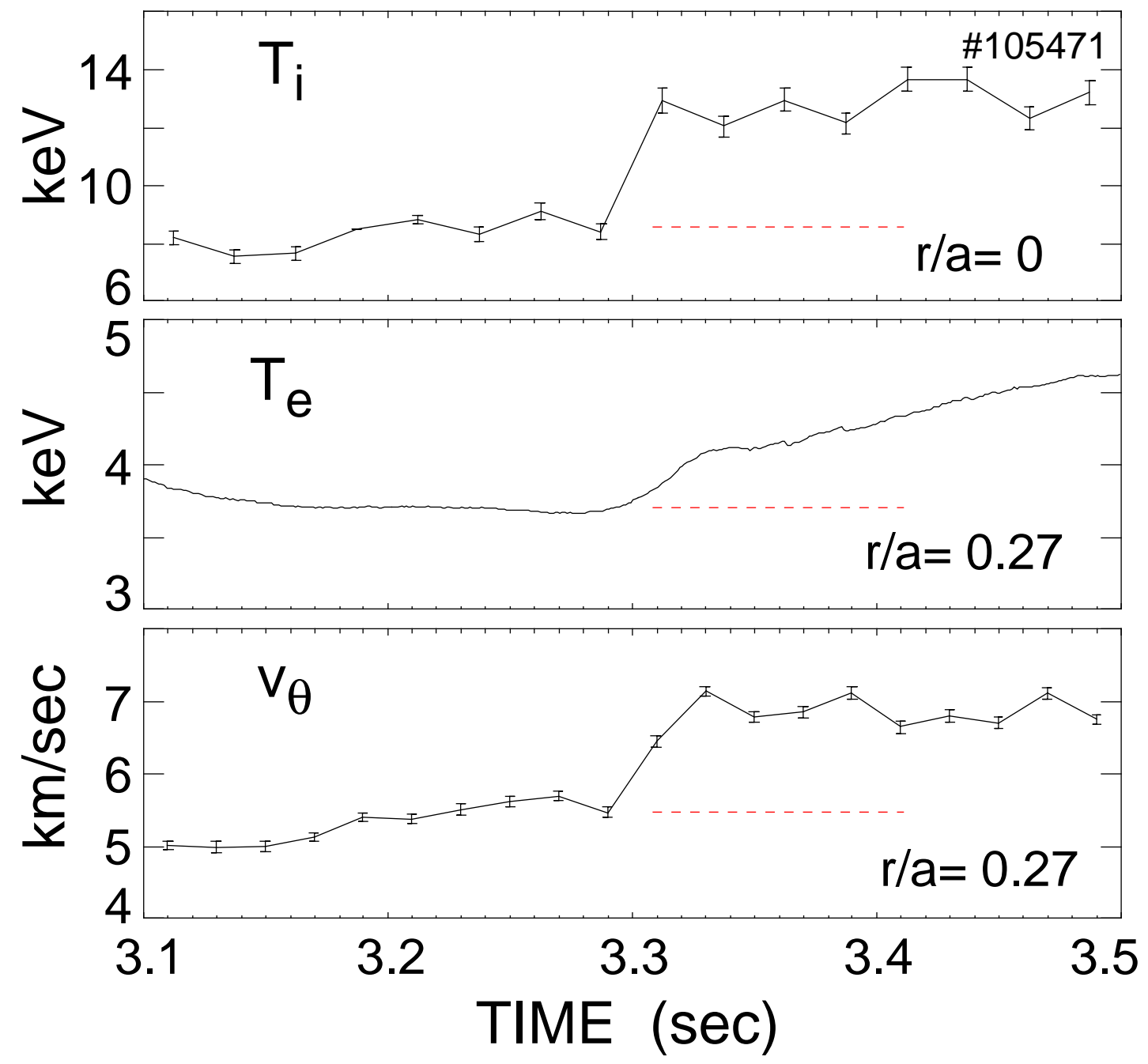

Fig. 4 The time behavior of $T_{i}$ and $T_{e}$ across the Type II ERS transition. No poloidal rotation change is observed before the transition. 